\title{
REMARKS ON FREE MUTUAL INFORMATION AND ORBITAL FREE ENTROPY
}

\author{
MASAKI IZUMI AND YOSHIMICHI UEDA
}

\begin{abstract}
The present notes provide a proof of $i^{*}(\mathbb{C} P+\mathbb{C}(I-P) ; \mathbb{C} Q+\mathbb{C}(I-$ $Q))=-\chi_{\text {orb }}(P, Q)$ for any pair of projections $P, Q$ with $\tau(P)=\tau(Q)=1 / 2$. The proof includes new extra observations, such as a subordination result in terms of Loewner equations. A study of the general case is also given.
\end{abstract}

\section{$\S 1$. Introduction}

There are two quantities which play a role of mutual information in free probability: one is the so-called free mutual information $i^{*}$ introduced by Voiculescu [22] in the late 1990s, and the other is the orbital free entropy $\chi_{\text {orb }}$ due to Hiai, Miyamoto, and the second-named author [13], [21] (and its new approaches $\tilde{\chi}_{\text {orb }}$, and so forth, due to Biane and Dabrowski [4]). These quantities have many properties in common, but no general relationship between them has been established so far. Any question about $i^{*}$ and/or $\chi_{\text {orb }}$ for two projections is known to be a "commutative one" in essence, that is, one that can essentially be handled within classical analysis (see [22, Section 12] and [14]), and a heuristic argument in [16, Section 3.2] supports that the identity $i^{*}=-\chi_{\text {orb }}$ holds at least for two projections. Hence the question of $i^{*}=-\chi_{\text {orb }}$ for two projections seems most tractable in this direction, and can be regarded as a counterpart of the single variable unification between two approaches $\chi$ and $\chi^{*}$ of free entropy, which was already established by Voiculescu (see [24]). Recently, Collins and Kemp [6] gave a proof of $i^{*}=-\chi_{\text {orb }}$ for two projections with $\tau(P)=\tau(Q)=1 / 2$ under a rather restricted assumption, along the lines of the above-mentioned heuristic argument (see [16] for details). Here we give an improved assertion of their result (i.e., completion of the analysis when $\tau(P)=\tau(Q)=1 / 2$ ) with a rather short and completely independent proof. Originally the first-named

Received August 19, 2013. Revised April 17, 2014. Accepted September 11, 2014. 2010 Mathematics Subject Classification. Primary 46L54; Secondary 94A17. 
author observed important ideas after the appearance of [16] as a preprint, and then we prepared an essential part of the present short notes some years ago (see, e.g., [21, Introduction]). Although the main theorem of the present notes is still an assertion about only the case of $\tau(P)=\tau(Q)=1 / 2$, a large part of its proof deals with any pair of projections and involves new extra observations which also enable us to give a partial result in the case of arbitrary trace values $\tau(P), \tau(Q)$. Hence these notes may have some degree of positive significance for future studies in this direction. We should also emphasize that the attempts are important as positive evidence for the conjecture that $i^{*}=-\chi_{\text {orb }}$ should hold for general random multivariables, though they have no direct connection with the unification conjecture for free entropy.

Throughout the present notes, let $(\mathcal{M}, \tau)$ denote a sufficiently large, tracial $W^{*}$-probability space with separable predual so that all the noncommutative random variables that we will deal with live in $(\mathcal{M}, \tau)$. The operator norm is denoted by $\|-\|_{\infty}$. Let $S_{t}, t \in[0, \infty)$, be a free additive Brownian motion in $(\mathcal{M}, \tau)$ (with $S_{0}=0$ ). A free unitary multiplicative Brownian motion $U_{t}, t \in[0, \infty)$, with $U_{0}=I$ introduced by Biane [1] is a noncommutative process consisting of unitary random variables determined by the free stochastic differential equation (free SDE for short) $d U_{t}=\sqrt{-1} d S_{t} U_{t}$ $(1 / 2) U_{t} d t, U_{0}=I$. For given two projections $P, Q$ in $\mathcal{M}$ that are freely independent of $\left\{U_{t}\right\}_{t \geq 0}$, the main objective here is to investigate the so-called liberation process $t \in[0, \infty) \mapsto\left(U_{t}(\mathbb{C} P+\mathbb{C}(I-P)) U_{t}^{*}, \mathbb{C} Q+\mathbb{C}(I-Q)\right)$ introduced by Voiculescu [22, Section 2] in relation with $i^{*}$ and $\chi_{\text {orb }}$. It is known that the liberation process can be understood by looking at the process of self-adjoint random variables $X_{t}:=Q U_{t} P U_{t}^{*} Q$. Thus we mainly investigate the process $X_{t}$ in what follows. One can easily derive the free SDE $d X_{t}=\Xi_{t} \sharp d S_{t}+Y_{t} d t$, where $\Xi_{t}:=\sqrt{-1}\left(Q \otimes U_{t} P U_{t}^{*} Q-Q U_{t} P U_{t}^{*} \otimes Q\right)$ and $Y_{t}:=\tau(P) Q-X_{t}$. (See [5] for definitions and notation concerning free SDEs such as $\sharp$-operation.) Note that $U_{t}$ is operator-norm continuous in $t$ by [1, Lemma 8], and so are $X_{t}, \Xi_{t}$, and $Y_{t}$ too.

\section{§2. Free SDE of $\left(z I-X_{t}\right)^{-1}$ and Cauchy transform of $X_{t}$}

Several ways to investigate the free SDE of the resolvent process $R(t, z):=$ $\left(z I-X_{t}\right)^{-1}$ and the Cauchy transform of $X_{t}$ have already been available (see, e.g., [8, Sections 6-7], [17, Section 3.2], [7, Section 3.1], and [6, Section 2]). However, we do give, for the reader's convenience, a simple proof 
of their explicit formulas by simple algebraic manipulations based on three naturally expected facts: (i) the free Itô formula, (ii) the resolvent process becomes again a "free Itô process," and (iii) every "free Itô process" has a unique "Doob-Meyer decomposition." In fact, the essential part of our proof will be done in several lines. Facts (i) and (ii) above were perfectly provided by Biane and Speicher [5], while (iii) is the latter half of Proposition 2.2 below. The proposition (with its lemma) is probably folklore.

Lemma 2.1. Let $\left\{\mathcal{M}_{t}\right\}_{t \geq 0}$ be an increasing filtration of von Neumann subalgebras of $\mathcal{M}$, and let $t \in[0, \infty) \mapsto K_{t}$ be a measurable process such that $K_{t} \in \mathcal{M}_{t}$ and $\sup _{0 \leq s \leq t}\left\|K_{s}\right\|_{\infty}<+\infty$ for all $t \geq 0$. If $t \in[0, \infty) \mapsto L_{t}:=$ $\int_{0}^{t} K_{s} d s$ defines a martingale adapted to $\left\{\mathcal{M}_{t}\right\}_{t \geq 0}$, then $L_{t}=0$ for all $t \geq 0$.

Proof. Since $L_{t}$ is a martingale, one has, for any division $0=: t_{0}<t_{1}<$ $\cdots<t_{n}:=t$

$$
\begin{aligned}
\tau\left(L_{t}^{*} L_{t}\right) & =\sum_{i=1}^{n} \tau\left(\left(L_{t_{i}}-L_{t_{i-1}}\right)^{*}\left(L_{t_{i}}-L_{t_{i-1}}\right)\right) \\
& \leq t\left(\sup _{0 \leq s \leq t}\left\|K_{s}\right\|_{\infty}\right)^{2} \sup _{1 \leq i \leq n}\left(t_{i}-t_{i-1}\right) .
\end{aligned}
$$

It follows that $L_{t}=0$, since $\sup _{1 \leq i \leq n}\left(t_{i}-t_{i-1}\right)$ can be arbitrarily small.

Proposition 2.2. Let $\left\{\mathcal{M}_{t}\right\}_{t \geq 0}$ be as in Lemma 2.1 such that $S_{t} \in \mathcal{M}_{t}$ for every $t \geq 0$. Let $t \in[0, \infty) \mapsto \Phi_{t}, \Phi_{t}^{\prime} \in \mathcal{M} \otimes_{\text {alg }} \mathcal{M}$ be operator-norm continuous biprocesses adapted to $\left\{\mathcal{M}_{t}\right\}_{t \geq 0}$, and let $t \in[0, \infty) \mapsto K_{t}, K_{t}^{\prime} \in \mathcal{M}$ be measurable processes such that $K_{t} \in \mathcal{M}_{t}$ and $\sup _{0 \leq s \leq t}\left\|K_{s}\right\|_{\infty}<+\infty$ for every $t \geq 0$, and the same holds for $K_{t}^{\prime}$. Then both $\bar{\Phi} \mathbf{1}_{[0, t]}$ and $\Phi^{\prime} \mathbf{1}_{[0, t]}$ fall in $\mathcal{B}_{\infty}^{a}$ (see [5, Section 2.1]) for every $t \geq 0$, and hence we have two free stochastic integrals $\int_{0}^{t} \Phi_{s} \sharp d S_{s}+\int_{0}^{t} K_{s} d s$ and $\int_{0}^{t} \Phi_{s}^{\prime} \sharp d S_{s}+\int_{0}^{t} K_{s}^{\prime} d s$ as in $[5$, Section 4.3] for every $t \geq 0$. If those free stochastic integrals define the same process, then $\Phi=\Phi^{\prime}$ and $K=K^{\prime}$ do almost surely in $t$.

Proof. The first part is trivial, and hence left to the reader. One has $\int_{0}^{t}\left(\Phi_{s}^{\prime}-\Phi_{s}\right) \sharp d S_{s}=\int_{0}^{t}\left(K_{s}-K_{s}^{\prime}\right) d s$, which must be zero by Lemma 2.1 and [5, Proposition 3.2.3]. Hence $\int_{0}^{t} \Phi_{s} \sharp d S_{s}=\int_{0}^{t} \Phi_{s}^{\prime} \sharp d S_{s}$ and $\int_{0}^{t} K_{s} d s=\int_{0}^{t} K_{s}^{\prime} d s$ hold for every $t>0$. The Itô isometry (see [5, Section 3.1]) immediately shows that $\Phi \mathbf{1}_{[0, t]}=\Phi^{\prime} \mathbf{1}_{[0, t]}$ holds in $\mathcal{B}_{2}^{a}$ for every $t>0$, and hence $\Phi=\Phi^{\prime}$ holds. Choose a dense countable subset $\left\{\varphi_{n}\right\}_{n \in \mathbb{N}}$ of the predual of $\mathcal{M}$. One has $\int_{t_{1}}^{t_{2}} \varphi_{n}\left(K_{s}-K_{s}^{\prime}\right) d s=0$ for every pair $0 \leq t_{1}<t_{2}<+\infty$ and $n \in \mathbb{N}$, which immediately implies that $K_{t}=K_{t}^{\prime}$ holds almost surely in $t$. 
One can choose, for each $z \in \mathbb{C}^{+}:=\{z \in \mathbb{C} \mid \operatorname{Im} z>0\}$, a rapidly decreasing function $f_{z}$ on $\mathbb{R}$ which coincides with $x \mapsto(z-x)^{-1}$ in a neighborhood of $[0,1]$, and thus $d R(t, z)=d\left(f_{z}\left(X_{t}\right)\right)=\left(\partial f_{z}\left(X_{t}\right) \sharp \Xi_{t}\right) \sharp d S_{t}+$ $\left(\partial f_{z}\left(X_{t}\right) \sharp Y_{t}+(1 / 2) \Delta_{\Xi_{t}} f_{z}\left(X_{t}\right)\right) d t$ holds by [5, Proposition 4.3.4]. Here we do not recall the definitions of $\partial f_{z}\left(X_{t}\right) \sharp \Xi_{t}, \partial f_{z}\left(X_{t}\right) \sharp Y_{t}$ and $\Delta_{\Xi_{t}} f_{z}\left(X_{t}\right)$ (those can be found in [5, Section 4.3], and we remark that $\left\|\Delta_{U} f(X)\right\|_{\infty}$ can be estimated by $\mathcal{I}_{2}(f)\|U\|_{\infty}^{2}$ in the same way as in the discussion following [5, Definition 4.1.1]). Here we need only the following trivial fact:

$$
\sup \left\{\left\|\partial f_{z}\left(X_{t}\right) \sharp Y_{t}\right\|_{\infty}+\left\|\Delta_{\Xi_{t}} f_{z}\left(X_{t}\right)\right\|_{\infty} \mid t \geq 0\right\}<+\infty .
$$

Write $M_{t}:=\int_{0}^{t}\left(\partial f_{z}\left(X_{s}\right) \sharp \Xi_{s}\right) \sharp d S_{s}, Z_{t}:=\partial f_{z}\left(X_{t}\right) \sharp Y_{t}+(1 / 2) \Delta_{\Xi_{t}} f_{z}\left(X_{t}\right)$, and $N_{t}:=\int_{0}^{t} \Xi_{s} \sharp d S_{s}$ for short, and let $z \in \mathbb{C}^{+}$be arbitrarily fixed. We have

$$
\begin{aligned}
0= & d\left(R(t, z)\left(z I-X_{t}\right)\right) \\
= & d R(t, z) \cdot\left(z I-X_{t}\right)+R(t, z) \cdot d\left(z I-X_{t}\right)-d M_{t} \cdot d N_{t} \\
= & d M_{t} \cdot\left(z I-X_{t}\right)+Z_{t}\left(z I-X_{t}\right) d t-R(t, z) \cdot d N_{t} \\
& -R(t, z) \cdot Y_{t} d t-d M_{t} \cdot d N_{t},
\end{aligned}
$$

and hence

$$
d M_{t} \cdot\left(z I-X_{t}\right)-R(t, z) \cdot d N_{t}=R(t, z) Y_{t} d t-Z_{t}\left(z I-X_{t}\right) d t+d M_{t} \cdot d N_{t} .
$$

This formal computation can easily be justified by the rigorous formulas in [5, Section 4.1]. Note that $d M_{t} \cdot d N_{t}=\left\langle\left\langle\partial f_{z}\left(X_{t}\right) \sharp \Xi_{t}, \Xi_{t}\right\rangle\right\rangle d t$ by the free Itô formula (see [5, Definition 4.1.1] for the precise definition of $\langle\langle-,-\rangle\rangle$ ). Therefore, Proposition 2.2 (which can be used thanks to (2.1)) shows that

$$
\begin{aligned}
& d M_{t}=R(t, z) \cdot d N_{t} \cdot R(t, z)=\left((R(t, z) \otimes R(t, z)) \sharp \Xi_{t}\right) \sharp d S_{t}, \\
& Z_{t} d t=R(t, z) Y_{t} R(t, z) d t+R(t, z) \cdot d N_{t} \cdot R(t, z) \cdot d N_{t} \cdot R(t, z) .
\end{aligned}
$$

It is easy to see, by the free Itô formula again, that $d N_{t} \cdot R(t, z) \cdot d N_{t}=\left(\tau(Q R(t, z)) X_{t}-2 \tau\left(X_{t} R(t, z)\right) X_{t}+\tau\left(X_{t} R(t, z)\right) Q\right) d t$, and hence (the first part of) the next proposition follows.

Proposition 2.3. For every $z \in \mathbb{C}^{+}$the resolvent process $R(t, z):=$ $\left(z I-X_{t}\right)^{-1}$ satisfies

$$
d R(t, z)=\left((R(t, z) \otimes R(t, z)) \sharp \Xi_{t}\right) \sharp d S_{t}+Z(t, z) d t,
$$


with

$$
\begin{aligned}
Z(t, z)= & \tau(P) R(t, z) Q R(t, z)-R(t, z) X_{t} R(t, z) \\
& -2 \tau\left(X_{t} R(t, z)\right) R(t, z) X_{t} R(t, z) \\
& +\tau(Q R(t, z)) R(t, z) X_{t} R(t, z) \\
& +\tau\left(X_{t} R(t, z)\right) R(t, z) Q R(t, z) .
\end{aligned}
$$

Moreover, the Cauchy transform $G(t, z):=\tau(R(t, z)), z \in \mathbb{C}^{+}$, satisfies the following partial differential equation (PDE for short):

$$
\frac{\partial G}{\partial t}=\frac{\partial}{\partial z}\left[\left(z^{2}-z\right) G^{2}+(2-\tau(P)-\tau(Q)-z) G-\frac{(1-\tau(P))(1-\tau(Q))}{z}\right] .
$$

Proof. The first part has already been obtained. Hence it suffices to show the desired PDE. Note that $Z_{t}=Z(t, z)$ is operator-norm continuous in $t$ thanks to the fact given at the end of Section 1. By the martingale property, $G(t, z)=\tau(R(t, z))=\tau(R(0, z))+\int_{0}^{t} \tau\left(Z_{s}\right) d s$, and hence, by (2.2),

$$
\begin{aligned}
\frac{\partial G}{\partial t}= & \tau\left(Z_{t}\right) \\
= & \tau(P) \tau\left(Q R(t, z)^{2}\right)-\tau\left(X_{t} R(t, z)^{2}\right)-2 \tau\left(X_{t} R(t, z)\right) \tau\left(X_{t} R(t, z)^{2}\right) \\
& +\tau(Q R(t, z)) \tau\left(X_{t} R(t, z)^{2}\right)+\tau\left(X_{t} R(t, z)\right) \tau\left(Q R(t, z)^{2}\right) .
\end{aligned}
$$

Note that $\tau\left(A R(t, z)^{2}\right)=-\frac{\partial}{\partial z} \tau(A R(t, z))$ for any $A \in \mathcal{M}$. Since $R(t, z)=$ $Q R(t, z) Q+z^{-1}(I-Q)$ and $I=\left(z I-X_{t}\right) R(t, z)=z R(t, z)-X_{t} R(t, z)$, we have $\tau(Q R(t, z))=G(t, z)-\frac{1-\tau(Q)}{z}$ and $\tau\left(X_{t} R(t, z)\right)=z G(t, z)-1$. These together imply the desired PDE.

\section{§3. Analysis of probability distribution of $X_{t}$}

Let $\nu_{t}$ be the probability distribution of $X_{t}$, that is, a unique probability measure on $[0,1]$ determined by $G(t, z)=\int_{[0,1]} \frac{1}{z-x} \nu_{t}(d x), z \in \mathbb{C}^{+}$. Define $c_{0}(t):=\tau\left(\left(I-U_{t} P U_{t}^{*}\right) \wedge(I-Q)+\left(I-U_{t} P U_{t}^{*}\right) \wedge Q+U_{t} P U_{t}^{*} \wedge(I-Q)\right)$, $c_{1}(t):=\tau\left(U_{t} P U_{t}^{*} \wedge Q\right), t \geq 0$. Several facts (see [22, Corollaries 1.7 and 8.6, Proposition 8.7, Lemma 12.5]) on liberation gradients with, for example, [16, (1.3)], together show that the projections $U_{t} P U_{t}^{*}, Q$ are in generic position for every $t>0$ and, moreover, that both $c_{0}(t)=1-\min \{\tau(P), \tau(Q)\}$ and $c_{1}(t)=\max \{\tau(P)+\tau(Q)-1,0\}$ hold for every $t>0$. (We will explain this fact in Remark 3.5 at the end of this section for the reader's convenience.) 
By a well-known fact (see, e.g., [12, Solution 122]) one easily sees that the functions $t \mapsto c_{i}(t)$ are upper semicontinuous, and hence $c_{0}(0) \geq c_{0}(+0)=$ $1-\min \{\tau(P), \tau(Q)\}$ and $c_{1}(0) \geq c_{1}(+0)=\max \{\tau(P)+\tau(Q)-1,0\}$.

Set $\mu_{t}:=\nu_{t}-(1-\min \{\tau(P), \tau(Q)\}) \delta_{0}-(\max \{\tau(P)+\tau(Q)-1,0\}) \delta_{1}$, $t \geq 0$, which defines a positive measure on $[0,1]$, since $c_{i}(0) \geq c_{i}(+0), i=0,1$. When $t>0, \mu_{t}$ agrees with the restriction of $\nu_{t}$ to $(0,1)$. Moreover, $\mu_{0}$ agrees with the restriction of $\nu_{0}$ to $(0,1)$ (or equivalently, both $c_{i}(0)=c_{i}(+0)$, $i=0,1$, hold) if and only if $P, Q$ are in generic position (see, e.g., the proof of [14, Theorem 3.2]). Denote by $F(t, z)$ the Cauchy transform of $\mu_{t}$ whose domain clearly contains $\mathbb{C} \backslash[0,1]$. A tedious computation derives the following PDE from Proposition 2.3:

$$
\frac{\partial F}{\partial t}=\frac{\partial}{\partial z}\left[\left(z^{2}-z\right) F^{2}+a(z-1) F+b z F\right]
$$

with $a:=|\tau(P)-\tau(Q)|$ and $b:=|\tau(P)+\tau(Q)-1|$.

Here, we use Geronimus's trick (see [11, Section 30]) based upon the socalled Szegö mapping; namely, we transform $z \in \mathbb{C} \backslash[0,1] \mapsto \zeta \in \mathbb{D}$, the open unit disk, by $z=\left(2+\zeta+\zeta^{-1}\right) / 4$ or $\zeta=2 z-1+2 \sqrt{z^{2}-z}$ (note that $\zeta \in \mathbb{D}$ determines the branch of $\sqrt{z^{2}-z}$ with a negative real value at $z=2$ ). Then we set $L(t, \zeta):=-\sqrt{z^{2}-z} F(t, z)$. Since $\frac{d \zeta}{d z}=\zeta / \sqrt{z^{2}-z}$, the PDE (3.1) becomes

$$
\frac{\partial L}{\partial t}+\zeta \frac{\partial}{\partial \zeta}\left[\left(L+a \frac{1-\zeta}{1+\zeta}+b \frac{1+\zeta}{1-\zeta}\right) L\right]=0
$$

Letting $\tilde{\mu}_{t}(d \theta)=\mu_{t}(d x)$ with $x=\cos ^{2}(\theta / 2)=\frac{1}{2}(1+\cos \theta), \theta \in[0, \pi]$, we have

$$
\begin{aligned}
L(t, \zeta) & =\frac{1}{4}\left(\frac{1}{\zeta}-\zeta\right) \int_{[0, \pi]} \frac{1}{\frac{1}{4}\left(2+\zeta+\frac{1}{\zeta}\right)-\cos ^{2}(\theta / 2)} \tilde{\mu}_{t}(d \theta) \\
& =\frac{1}{4}\left(\frac{1}{\zeta}-\zeta\right) \int_{[0, \pi]} \frac{1}{\frac{1}{4}\left(2+\zeta+\frac{1}{\zeta}\right)-\frac{1}{4}\left(2+e^{\sqrt{-1} \theta}+e^{-\sqrt{-1} \theta}\right)} \tilde{\mu}_{t}(d \theta) \\
& =\int_{[0, \pi]}\left(-1+\frac{e^{\sqrt{-1} \theta}}{e^{\sqrt{-1} \theta}-\zeta}+\frac{e^{-\sqrt{-1} \theta}}{e^{-\sqrt{-1} \theta}-\zeta}\right) \tilde{\mu}_{t}(d \theta),
\end{aligned}
$$

and thus the symmetrization $\hat{\mu}_{t}:=\frac{1}{2}\left(\tilde{\mu}_{t}+\left(\tilde{\mu}_{t} \uparrow(0, \pi)\right) \circ j^{-1}\right)$ with $j: \theta \in$ $(0, \pi) \mapsto-\theta \in(-\pi, 0)$ satisfies

$$
L(t, \zeta)=\int_{(-\pi, \pi]} \frac{e^{\sqrt{-1} \theta}+\zeta}{e^{\sqrt{-1} \theta}-\zeta} \hat{\mu}_{t}(d \theta) .
$$


Define $H(t, \zeta):=\left(L(t, \zeta)+a \frac{1-\zeta}{1+\zeta}+b \frac{1+\zeta}{1-\zeta}\right) L(t, \zeta)$, and by (3.2), we have

$$
\frac{\partial H}{\partial t}+\zeta\left(2 L(t, \zeta)+a \frac{1-\zeta}{1+\zeta}+b \frac{1+\zeta}{1-\zeta}\right) \frac{\partial H}{\partial \zeta}=0 .
$$

As usual, let us consider the ordinary differential equations (ODEs for short) of characteristic curve $t \mapsto\left(g_{t}(\zeta), u_{t}(\zeta):=H\left(t, g_{t}(\zeta)\right)\right)$ associated with the PDE (3.4):

$$
\begin{aligned}
& \dot{g}_{t}(\zeta)=g_{t}(\zeta)\left[2 L\left(t, g_{t}(\zeta)\right)+a \frac{1-g_{t}(\zeta)}{1+g_{t}(\zeta)}+b \frac{1+g_{t}(\zeta)}{1-g_{t}(\zeta)}\right], \quad g_{0}(\zeta)=\zeta \\
& \dot{u}_{t}(\zeta)=0, \quad u_{0}(\zeta)=H(0, \zeta)
\end{aligned}
$$

Here the dot symbol (') denotes the differentiation in $t$. The ODE (3.5) is nothing less than the radial Loewner (or Löwner-Kufarev) equation (or more precisely, radial Loewner $O D E$ ) determined by the 1-parameter family of measures $t \mapsto 2 \hat{\mu}_{t}+a \delta_{\pi}+b \delta_{0}$. Note by, for example, [16, (1.3)], that $2 \hat{\mu}_{t}+a \delta_{\pi}+b \delta_{0}$ defines a probability measure on $\mathbb{T}=(-\pi, \pi]$ for every $t \geq 0$. (This follows from the facts that $U_{t} P U_{t}^{*}, Q$ are in generic position for every $t>0$ as remarked before and that $\hat{\mu}_{t} \rightarrow \hat{\mu}_{0}$ weakly as $t \searrow 0$.) Thus, by a standard fact (see, e.g., [20, Theorem 4.14]), the radial Loewner ODE (3.5) defines a unique 1-parameter family of conformal transformations $g_{t}: \mathbb{D}_{t}:=$ $\left\{\zeta \in \mathbb{D} \mid T_{\zeta}>t\right\} \rightarrow \mathbb{D}$ with $g_{t}(0)=0$ and $g_{t}^{\prime}(0)=e^{t}$ (the prime symbol $\left(^{\prime}\right)$ denotes the differentiation in $\zeta$ ), where $T_{\zeta}, \zeta \in \mathbb{D}$, is the supremum of all $T$ such that a solution of (3.5) exists until time $T$ in such a way that $g_{t}(\zeta) \in \mathbb{D}$ holds for every $t \leq T$. It is known (see, e.g., [20, Remark 4.15]) that the inverse $f_{t}:=g_{t}^{-1}: \mathbb{D} \rightarrow \mathbb{D}_{t}$ satisfies

$$
\dot{f}_{t}(\zeta)=-\zeta f_{t}^{\prime}(\zeta)\left[\int_{(-\pi, \pi]} \frac{e^{\sqrt{-1} \theta}+\zeta}{e^{\sqrt{-1} \theta}-\zeta}\left(2 \hat{\mu}_{t}+a \delta_{\pi}+b \delta_{0}\right)(d \theta)\right], \quad f_{0}(\zeta)=\zeta
$$

a radial Loewner PDE. The ODE (3.6) shows that $H\left(t, g_{t}(\zeta)\right)=u_{t}(\zeta)=$ $u_{0}(\zeta)=H(0, \zeta)$, and hence $H(t, \zeta)=H\left(0, f_{t}(\zeta)\right)$ holds for every $\zeta \in \mathbb{D}$. This implies that

$$
\begin{aligned}
L(t, \zeta)= & -\frac{1}{2}\left(a \frac{1-\zeta}{1+\zeta}+b \frac{1+\zeta}{1-\zeta}\right) \\
& +\frac{1}{2} \sqrt{\left(a \frac{1-\zeta}{1+\zeta}+b \frac{1+\zeta}{1-\zeta}\right)^{2}+4 H\left(0, f_{t}(\zeta)\right)}
\end{aligned}
$$


where $\sqrt{-}$ is the principal branch. The discussions so far are summarized as follows.

Proposition 3.1. Let $\nu_{t}$ be the probability distribution of $X_{t}$.

The positive measure $\mu_{t}:=\nu_{t}-(1-\min \{\tau(P), \tau(Q)\}) \delta_{0}-(\max \{\tau(P)+$ $\tau(Q)-1,0\}) \delta_{1}$ coincides with the restriction of $\nu_{t}$ to $(0,1)$ for every $t>0$, and moreover, does so also at $t=0$ (or equivalently, $\mu_{0}$ has no atom at both 0 and 1), if and only if the given two projections $P, Q$ are in generic position.

Define the positive measure $\tilde{\mu}_{t}(d \theta):=\mu_{t}(d x)$ on $[0, \pi]$ via $x=\cos ^{2}(\theta / 2)$, and consider $L(t, \zeta):=\int_{(-\pi, \pi)} \frac{e^{\sqrt{-1} \theta}+\zeta}{e^{\sqrt{-1} \theta}-\zeta} \hat{\mu}_{t}(d \theta), \zeta \in \mathbb{D}$, obtained from the symmetrization $\hat{\mu}_{t}:=\frac{1}{2}\left(\tilde{\mu}_{t}+\left(\tilde{\mu}_{t} \uparrow_{(0, \pi)}\right) \circ j^{-1}\right)$ with the mapping $j: \theta \in(0, \pi) \mapsto$ $-\theta \in(-\pi, 0)$. Then the unique 1-parameter, subordinate family of conformal self-maps $f_{t}$ on $\mathbb{D}$ obtained from the radial Loewner PDE (3.7) driven by the probability measures $2 \hat{\mu}_{t}+a \delta_{\pi}+b \delta_{0}$ gives the subordination relation $H(t, \zeta)=H\left(0, f_{t}(\zeta)\right)$, where $H(t, \zeta):=\left(L(t, \zeta)+a \frac{1-\zeta}{1+\zeta}+b \frac{1+\zeta}{1-\zeta}\right) L(t, \zeta)$ with $a=|\tau(P)-\tau(Q)|$ and $b=|\tau(P)+\tau(Q)-1|$.

The next corollary is a specialization of the above proposition.

Corollary 3.2. Let $L(t, \zeta), f_{t}(\zeta)$ be as in Proposition 3.1, set $g_{t}(\zeta):=$ $f_{t}^{-1}(\zeta)$, and suppose that $\tau(P)=\tau(Q)=1 / 2$ or equivalently that $a=b=0$. Then

- $L(t, \zeta)=L\left(0, f_{t}(\zeta)\right)$, that is, $L(t, \zeta)$ is subordinate to $L(s, \zeta)$ for $s<t$,

- $g_{t}(\zeta)=\zeta e^{2 t L(0, \zeta)}$ and $f_{t}(\zeta)=\zeta e^{-2 t L(t, \zeta)}$,

- $\operatorname{Re} L(t, \zeta)=\left(\log |\zeta|-\log \left|f_{t}(\zeta)\right|\right) / 2 t, t>0$ and $\zeta \in \mathbb{D} \backslash\{0\}$.

Proof. Under the assumption here, the subordination relation in Proposition 3.1 turns out to be the exact subordination $L(t, \zeta)=L\left(0, f_{t}(\zeta)\right)$. This together with (3.5) implies that $\dot{g}_{t}(\zeta)=2 g_{t}(\zeta) L\left(t, g_{t}(\zeta)\right)=2 g_{t}(\zeta) L(0, \zeta)$. This ODE can easily be solved as $g_{t}(\zeta)=\zeta e^{2 t L(0, \zeta)}$, implying $\zeta=$ $f_{t}(\zeta) e^{2 t L\left(0, f_{t}(\zeta)\right)}=f_{t}(\zeta) e^{2 t L(t, \zeta)}$. The final assertion immediately follows.

This allows us to prove some properties of $\hat{\mu}_{t}$ by analyzing $f_{t}(\zeta)$ and/or $g_{t}(\zeta)$ when $\tau(P)=\tau(Q)=1 / 2$, but we give a more useful observation as the next proposition. The proposition immediately follows from only (3.2) and (3.3). This means that the proof of the main result of the present notes (Theorem 4.3) needs only a few pages. 
Proposition 3.3. Under the same assumption as in Corollary 3.2, $\left\{2 \hat{\mu}_{t / 2}\right\}_{t \geq 0}$ is identical to the 1-parameter semigroup of probability distributions associated with a free unitary multiplicative Brownian motion with initial distribution $2 \hat{\mu}_{0}$.

Proof. Since $\hat{\mu}_{t}$ is symmetric, we have

$$
\psi(t, \zeta):=\int_{(-\pi, \pi]} \frac{\zeta e^{\sqrt{-1} \theta}}{1-\zeta e^{\sqrt{-1} \theta}}\left(2 \hat{\mu}_{t / 2}\right)(d \theta)=L(t / 2, \zeta)-1 / 2,
$$

the moment generating function of the measure $2 \hat{\mu}_{t / 2}$. The PDE (3.2) can easily be transformed into

$$
\dot{\psi}+\zeta(\psi+1 / 2) \psi^{\prime}=0
$$

This is the PDE that the moment generating function of a free unitary multiplicative Brownian motion satisfies (see, e.g., the proof of [22, Proposition 10.8]), and hence the desired assertion follows as seen below. Let $U$ be a unitary random variable with distribution $2 \hat{\mu}_{0}$, which is freely independent of $\left\{U_{t}\right\}_{t \geq 0}$. Set $\tilde{\psi}(t, \zeta):=\tau\left(\left(I-\zeta U_{t} U\right)^{-1}-I\right), \zeta \in \mathbb{D}$, the moment generating function of $U_{t} U$. Then $\tilde{\psi}$ satisfies the same PDE (3.9). Write $\psi(t, \zeta)=$ $\sum_{n=1}^{\infty} c_{n}(t) \zeta^{n}, \tilde{\psi}(t, \zeta)=\sum_{n=1}^{\infty} \tilde{c}_{n}(t) \zeta^{n}$. Developing (3.9) into power series as above, we see that both the coefficients $c_{n}$ and $\tilde{c}_{n}$ must satisfy that $\dot{f}_{1}=$ $-\frac{1}{2} f_{1}, \dot{f}_{n}=-\frac{n}{2} f_{n}-\sum_{k=1}^{n-1} k f_{k} f_{n-k}(n=2,3, \ldots)$ with $f_{n}=c_{n}$ or $\tilde{c}_{n}$. Since $\psi(0, \zeta)=\int_{(-\pi, \pi]} \frac{\zeta e^{\sqrt{-1} \theta}}{1-\zeta e^{\sqrt{-1} \theta}}\left(2 \hat{\mu}_{0}\right)(d \theta)=\tilde{\psi}(0, \zeta), \zeta \in \mathbb{D}$, one has $c_{n}(0)=\tilde{c}_{n}(0)$ for every $n$. Hence one can recursively show that $\int_{(-\pi, \pi]} e^{\sqrt{-1} n \theta}\left(2 \hat{\mu}_{t / 2}\right)(d \theta)=$ $c_{n}(t)=\tilde{c}_{n}(t)=\tau\left(\left(U_{t} U\right)^{n}\right)$.

Remarks 3.4. (1) The above proposition enables us to derive detailed information about $\mu_{t}$ from many existing results (see [1], [2, Section 4.2], [22, Section 1]) on free unitary multiplicative Brownian motions (with the help of $S$-transform machinery; see, e.g., [23, Section 3]) when $\tau(P)=\tau(Q)=1 / 2$. Moreover, the recent work [25] generalizing Biane's analysis in [2, Section 4.2] gives more detailed properties of $\hat{\mu}_{t}$ and hence those of $\mu_{t}$, though we omit giving further results in this direction here.

(2) The above proposition also recaptures, as its specialization, Corollary 2 of [9]. In fact, the free Jacobi process with parameter $(\lambda, \theta)=(1,1 / 2)$ (see [8]) is exactly our $X_{t}$ (viewed as a random variable in $\left.\left(Q \mathcal{M} Q, \frac{1}{\tau(Q)} \tau\right)\right)$ with $P=Q$ and $\tau(P)=\tau(Q)=1 / 2$. Hence the initial distribution $2 \hat{\mu}_{0}$ is the unit mass at $\theta=0$, and thus the probability distribution 
of the free Jacobi process with parameter $(\lambda, \theta)=(1,1 / 2)$ is exactly that of the free unitary multiplicative Brownian motion via $x=\cos ^{2}(\theta / 2)$.

REMARK 3.5. The following simple "liberation-theoretic" proof of the fact that $U_{t} P U_{t}^{*}, Q$ are in generic position for every $t>0$ has been available so far. By [22, Corollary 1.7, Proposition 8.7], $d_{U_{t}: \mathbb{C}^{1}}^{1 \otimes 1}$ (see the notation there) exists in $L^{2}$ for every $t>0$, which implies, by [22, Corollary 8.6], that so does the liberation gradient $j\left(U_{t}(\mathbb{C} P+\mathbb{C}(I-P)) U_{t}^{*}: \mathbb{C} Q+\mathbb{C}(I-Q)\right)$. Therefore, by [22, Lemma 12.5] (together with $U_{t}(\mathbb{C} P+\mathbb{C}(I-P)) U_{t}^{*}=\mathbb{C} U_{t} P U_{t}^{*}+$ $\left.\mathbb{C}\left(I-U_{t} P U_{t}^{*}\right)\right)$, we conclude that $U_{t} P U_{t}^{*}, Q$ are in generic position for every $t>0$. This argument indeed shows the following stronger result: $U P U^{*}, Q$ are in generic position for any unitary $U$ with finite Fisher information $F(U)<+\infty$ (see [22, Definition 8.9]) which is freely independent of $P, Q$.

\section{§4. Free mutual information and orbital free entropy}

To a given pair of projections $P, Q$ we can associate four quantities: the liberation gradient, the liberation Fisher information, the mutual free information of $\mathbb{C} P+\mathbb{C}(I-P)$ relative to $\mathbb{C} Q+\mathbb{C}(I-Q)$ denoted by $j(\mathbb{C} P+$ $\mathbb{C}(I-P): \mathbb{C} Q+\mathbb{C}(I-Q))(=: j(P: Q)$ for short $), \varphi^{*}(\mathbb{C} P+\mathbb{C}(I-P): \mathbb{C} Q+$ $\mathbb{C}(I-Q))\left(=: \varphi^{*}(P: Q)\right), i^{*}(\mathbb{C} P+\mathbb{C}(I-P): \mathbb{C} Q+\mathbb{C}(I-Q))\left(=: i^{*}(P: Q)\right)$, respectively, all of which are due to Voiculescu [22], and the orbital free entropy $\chi_{\mathrm{orb}}(P, Q)$ (see [13]). The free mutual information $i^{*}(\mathbb{C} P+\mathbb{C}(I-P)$; $\mathbb{C} Q+\mathbb{C}(I-Q))$ of $\mathbb{C} P+\mathbb{C}(I-P)$ and $\mathbb{C} P+\mathbb{C}(I-P)$ coincides with the above $i^{*}(P: Q)$ (see [22, Remarks 10.2(c)]), and hence it suffices to compute $i^{*}(P: Q)$ for our purpose. According to the change of variables $\mu_{t} \rightsquigarrow \tilde{\mu}_{t} \rightsquigarrow \hat{\mu}_{t}$ in Section 3, we need to reformulate Voiculescu's computation of $\varphi^{*}(P: Q)$ (see [22, Section 12]), as well as the previous computation of $\chi_{\text {orb }}(P, Q)$ essentially due to Hiai and Petz in [14, Proposition 3.3] (see also [13, p. 236], [15, Section 2]).

For simplicity, write $\delta:=\delta_{\mathbb{C} P+\mathbb{C}(I-P): \mathbb{C} Q+\mathbb{C}(I-Q)}$, the derivation of $\mathbb{C} P+$ $\mathbb{C}(I-P)$ relative to $\mathbb{C} Q+\mathbb{C}(I-Q)$ (see [22, Section 5.3]). Let $\mu$ be the restriction of the probability distribution of $Q P Q$ to $(0,1)$. Note that the measure $\mu$ is not changed if $Q P Q$ is replaced with $P Q P$ and that $\mu$ is exactly $\frac{1}{2} \nu$ in [22, Section 12]. The proof of Lemma 4.1 below uses the following well-known representation of $P, Q$. Set $E_{00}:=(I-P) \wedge(I-Q)$, $E_{10}:=P \wedge(I-Q), E_{01}:=(I-P) \wedge Q, E_{11}:=P \wedge Q$, which are minimal and central projections of the von Neumann subalgebra $W^{*}(P, Q)$ generated by $P, Q$. With $E:=I-\sum_{i, j=0,1} E_{i j}$ the central summand $W^{*}(P, Q) E$ is 
identified with $M_{2}\left(L^{\infty}((0,1), \mu)\right)$ by

$$
P E=\left[\begin{array}{ll}
1 & 0 \\
0 & 0
\end{array}\right], \quad Q E=\left[\begin{array}{cc}
x & \sqrt{x(1-x)} \\
\sqrt{x(1-x)} & 1-x
\end{array}\right] \quad(x \in(0,1)) .
$$

With this identification $W^{*}(P, Q) E=M_{2}\left(L^{\infty}((0,1), \mu)\right)$ the tracial state $\tau$ becomes

$$
\tau\left(\left[f_{i j}\right]\right)=\int_{(0,1)}\left(f_{11}(x)+f_{22}(x)\right) \mu(d x), \quad\left[f_{i j}\right] \in M_{2}\left(L^{\infty}((0,1), \mu)\right) .
$$

Write $a:=|\tau(P)-\tau(Q)|$ and $b:=|\tau(P)+\tau(Q)-1|$ for simplicity. If $P, Q$ are in generic position, then by [22, Sections 12.1-12.6] one has, for $n \geq 1$,

$$
\begin{aligned}
(\tau \otimes & \tau) \circ \delta(P Q)^{n} \\
= & 2 \mathrm{PV} \iint_{(0,1)^{2}} x^{n}(x-1) \frac{1}{x-y} \mu \otimes \mu(d x, d y) \\
& +(a+b) \int_{(0,1)} x^{n-1}(x-1) \mu(d x)+b \int_{(0,1)} x^{n-1} \mu(d x) .
\end{aligned}
$$

Here PV is the sign of the Cauchy principal value. With $\theta \in(0, \pi) \mapsto x=$ $\cos ^{2}(\theta / 2) \in(0,1)$ and $\tilde{\mu}(d \theta):=\mu(d x)$ as in Section 3 , we have, for $n \geq 1$,

$$
\begin{aligned}
& (\tau \otimes \tau) \circ \delta(P Q)^{n} \\
& (4.1)=-2 \mathrm{PV} \iint_{(0, \pi)^{2}} \cos ^{2 n-1}(\alpha / 2) \sin (\alpha / 2) \frac{\sin \alpha}{\cos \alpha-\cos \beta} \tilde{\mu} \otimes \tilde{\mu}(d \alpha, d \beta) \\
& -a \int_{(0, \pi)} \cos ^{2(n-1)}(\theta / 2) \sin ^{2}(\theta / 2) \tilde{\mu}(d \theta)+b \int_{(0, \pi)} \cos ^{2 n}(\theta / 2) \tilde{\mu}(d \theta) .
\end{aligned}
$$

Here we further suppose that $\mu$ has a density function $h$, that is, $\mu(d x)=$ $h(x) d x$. Set $\tilde{h}(\theta):=h\left(\cos ^{2}(\theta / 2)\right) \sin (\theta / 2) \cos (\theta / 2)$, and thus $\tilde{\mu}(d \theta)=\tilde{h}(\theta) d \theta$. Then the symmetrization $\hat{\mu}:=\frac{1}{2}\left(\tilde{\mu}+\tilde{\mu} \circ j^{-1}\right)$ with $j: \theta \in(0, \pi) \mapsto-\theta \in$ $(-\pi, 0)$ also has a density function, that is, $\hat{\mu}(d \theta)=\hat{h}(\theta) d \theta$ with $\hat{h}(\theta)=$ $\left(h\left(\cos ^{2}(\theta / 2)\right)|\sin \theta|\right) / 4=\left(h\left(\cos ^{2}(\theta / 2)\right)|\sin (\theta / 2)| \cos (\theta / 2)\right) / 2, \quad \theta \in(-\pi, \pi)$. (Note that we use the nonnormalized Lebesgue measure $d \theta$ as the reference measure on $\mathbb{T}=(-\pi, \pi]$ following Koosis's book [19], in contrast with [22].) The Hilbert transform (or the harmonic conjugate) of $\hat{h}$ is defined by 


$$
(H \hat{h})(\theta):=\frac{1}{2 \pi} \mathrm{PV} \int \frac{\hat{h}(\phi)}{\tan ((\theta-\phi) / 2)} d \phi, \quad \theta \in \mathbb{T}=(-\pi, \pi],
$$

which exists almost everywhere (see [19, Section III.C.2]). As in [18, Section $6.7,(6.86)]$, the restriction of $H \hat{h}$ to $(0, \pi)$ can be rewritten in terms of $\tilde{h}$ as

$$
(H \hat{h})(\theta)=-\frac{\sin \theta}{2 \pi} \mathrm{PV} \int_{(0, \pi)} \frac{\tilde{h}(\phi)}{\cos \theta-\cos \phi} d \phi, \quad \theta \in(0, \pi) .
$$

Under the equivalent assumptions

$$
\begin{aligned}
& \hat{h} \in L^{2}(-\pi, \pi) \text { if and only if } \tilde{h} \in L^{2}(0, \pi), \\
& \quad \text { or equivalently } \int_{(0,1)} \sqrt{x(1-x)} h(x)^{2} d x<+\infty,
\end{aligned}
$$

the Cauchy principal value in $(4.2)$ converges in $L^{2}$-norm by $[19$, Section I.E.4]. Define a function $\xi:(0, \pi) \rightarrow M_{2}(\mathbb{C})$ by

$$
\xi(\theta):=(4 \pi(H \hat{h})(\theta)-a \tan (\theta / 2)+b \cot (\theta / 2))\left[\begin{array}{cc}
0 & -1 \\
1 & 0
\end{array}\right],
$$

which is affiliated with $M_{2}\left(L^{\infty}((0,1), \mu)\right)=W^{*}(P, Q) E$ via $x=\cos ^{2}(\theta / 2)$. With these preliminaries, we have the following.

Lemma 4.1. Assume that $P, Q$ are in generic position. If $\mu(d x)=h(x) d x$ such that $h$ satisfies (4.3), then $\xi$ gives the liberation gradient $j(P: Q)$ as long as $\theta \mapsto 4 \pi(H \hat{h})(\theta)-a \tan (\theta / 2)+b \cot (\theta / 2)$ is integrable with respect to $\tilde{\mu}$, and moreover,

$$
\begin{aligned}
\varphi^{*}(P: Q) & =\int_{(0, \pi)} 2|4 \pi(H \hat{h})(\theta)-a \tan (\theta / 2)+b \cot (\theta / 2)|^{2} \tilde{\mu}(d \theta) \\
& =\int_{(-\pi, \pi)}|2 \pi(H(2 \hat{h}))(\theta)-a \tan (\theta / 2)+b \cot (\theta / 2)|^{2}(2 \hat{\mu})(d \theta)
\end{aligned}
$$

possibly to be $+\infty$ under the same integrability assumption.

Proof. By the computation (4.1) together with (4.2) and the hypotheses (4.3), one can easily see that $\tau\left(\xi(P Q)^{n}\right)=(\tau \otimes \tau) \circ \delta(P Q)^{n}$ for $n \geq 1$ (whose proof is just a translation of the proof of [22, Proposition 12.7] into the present context). Hence one gets $j(P: Q)=\xi$ by its definition (see [22, Definition 5.4]) under the integrability assumption. Then the first equality 
in (4.4) is immediate, and the second one follows from the fact that $\theta \mapsto$ $4 \pi(H \hat{h})(\theta)-a \tan (\theta / 2)+b \cot (\theta / 2)$ is an odd function.

Keep the symbols $\mu, \tilde{\mu}, \hat{\mu}$, and $a, b$ above. If $P, Q$ are in generic position, then

$$
\begin{aligned}
\chi_{\mathrm{orb}}(P, Q)= & \iint_{(0,1)^{2}} \log |x-y| \mu \otimes \mu(d x, d y) \\
& +a \int_{(0,1)} \log x \mu(d x)+b \int_{(0,1)} \log (1-x) \mu(d x)+C ;
\end{aligned}
$$

otherwise $\chi_{\mathrm{orb}}(P, Q)=-\infty$, where $C$ is a unique constant determined by $\chi_{\mathrm{orb}}(P, Q)=0$ when $P, Q$ are freely independent and keep the prescribed values $\tau(P), \tau(Q)$. In particular, $C=(\log 2) / 2$ when $\tau(P)=\tau(Q)=1 / 2$ (see, e.g., [16, Lemma 1.1], [13, Lemma 2.4]). In what follows, we assume that $P, Q$ are in generic position, and, in particular, $\mu((0,1))=(1-a-b) / 2$ by [16, (1.3)]. Since $|\cos \alpha-\cos \beta|=\left(\left|e^{\sqrt{-1} \alpha}-e^{\sqrt{-1} \beta}\right| \cdot\left|e^{\sqrt{-1} \alpha}-e^{-\sqrt{-1} \beta}\right|\right) / 2$, we have, with $(x, y)=\left(\cos ^{2}(\alpha / 2), \cos ^{2}(\beta / 2)\right)$,

$$
\begin{aligned}
& \iint_{(0,1)^{2}} \log |x-y| \mu \otimes \mu(d x, d y) \\
& =\iint_{(0, \pi)^{2}}(\log |\cos \alpha-\cos \beta|-\log 2) \tilde{\mu} \otimes \tilde{\mu}(d \alpha, d \beta) \\
& =\iint_{(0, \pi)^{2}}\left(\log \left|e^{\sqrt{-1} \alpha}-e^{\sqrt{-1} \beta}\right|+\log \left|e^{\sqrt{-1} \alpha}-e^{-\sqrt{-1} \beta}\right|-2 \log 2\right) \\
& \quad \times \tilde{\mu} \otimes \tilde{\mu}(d \alpha, d \beta) \\
& =2 \iint_{(-\pi, \pi)^{2}} \log \left|e^{\sqrt{-1} \alpha}-e^{\sqrt{-1} \beta}\right| \hat{\mu} \otimes \hat{\mu}(d \alpha, d \beta)-\frac{\log 2}{2}(1-a-b)^{2} .
\end{aligned}
$$

Here we used the fact that $\mu(0,1)=\tilde{\mu}(0, \pi)=\hat{\mu}(-\pi, \pi)=(1-a-b) / 2$. We also have, with $x=\cos ^{2}(\theta / 2)$,

$$
\begin{aligned}
\int_{(0,1)} \log x \mu(d x) & =2 \int_{(-\pi, \pi)} \log \left|1+e^{\sqrt{-1} \theta}\right| \hat{\mu}(d \theta)-(1-a-b) \log 2, \\
\int_{(0,1)} \log (1-x) \mu(d x) & =2 \int_{(-\pi, \pi)} \log \left|1-e^{\sqrt{-1} \theta}\right| \hat{\mu}(d \theta)-(1-a-b) \log 2 .
\end{aligned}
$$

Therefore, we conclude the following. 
LEMMA 4.2. If $P, Q$ are in generic position, then

$$
\begin{aligned}
\chi_{\mathrm{orb}}(P, Q)= & 2\left\{\iint_{(-\pi, \pi)^{2}} \log \left|e^{\sqrt{-1} \alpha}-e^{\sqrt{-1} \beta}\right| \hat{\mu} \otimes \hat{\mu}(d \alpha, d \beta)\right. \\
& +a \int_{(-\pi, \pi)} \log \left|1+e^{\sqrt{-1} \theta}\right| \hat{\mu}(d \theta) \\
& \left.+b \int_{(-\pi, \pi)} \log \left|1-e^{\sqrt{-1} \theta}\right| \hat{\mu}(d \theta)\right\}+Z
\end{aligned}
$$

with a universal constant $Z=Z_{\tau(P), \tau(Q)}$ depending only on $\tau(P), \tau(Q)$; otherwise $\chi_{\mathrm{orb}}(P, Q)=-\infty$. In particular, if $\tau(P)=\tau(Q)=1 / 2$, then the above formula of $\chi_{\mathrm{orb}}(P, Q)$ simply becomes

$$
\chi_{\mathrm{orb}}(P, Q)=2 \iint_{(-\pi, \pi)^{2}} \log \left|e^{\sqrt{-1} \alpha}-e^{\sqrt{-1} \beta}\right| \hat{\mu} \otimes \hat{\mu}(d \alpha, d \beta) .
$$

Let us return to the original situation; thus we use the notation in Section 3. We can now reduce our question to [22, Corollary 10.9] when $\tau(P)=$ $\tau(Q)=1 / 2$.

Theorem 4.3. For any two projections $P, Q$ with $\tau(P)=\tau(Q)=1 / 2$, one has

$i^{*}(\mathbb{C} P+\mathbb{C}(I-P) ; \mathbb{C} Q+\mathbb{C}(I-Q))=-\chi_{\text {orb }}(P, Q)$ possibly with $+\infty=+\infty$.

Proof. By Proposition 3.3 and [22, Corollary 1.7], $2 \hat{\mu}_{t / 2}$ has an $L^{\infty}$ density $2 \hat{h}(t / 2, \theta)$ (with respect to $d \theta$ rather than $d \theta / 2 \pi$ as in [22]) for every $t>0$. By [22, Corollary 10.9], we have

$$
\begin{aligned}
& -\iint_{(-\pi, \pi]^{2}} \log \left|e^{\sqrt{-1} \alpha}-e^{\sqrt{-1} \beta}\right|\left(2 \hat{\mu}_{0}\right) \otimes\left(2 \hat{\mu}_{0}\right)(d \alpha, d \beta) \\
& =\frac{1}{2} \int_{0}^{+\infty} \int_{(-\pi, \pi]}(2 \pi H(2 \hat{h}(t / 2,-))(\theta))^{2} 2 \hat{h}(t / 2, \theta) d \theta d t .
\end{aligned}
$$

By Lemma 4.1 (together with Remark 3.5),

$$
\int_{(-\pi, \pi)}(2 \pi H(2 \hat{h}(t / 2,-))(\theta))^{2} 2 \hat{h}(t / 2, \theta) d \theta=\varphi^{*}\left(U_{t / 2} P U_{t / 2}^{*}: Q\right)
$$

holds for every $t>0$ so that the right-hand side of (4.5) is identical to

$$
\frac{1}{2} \int_{0}^{+\infty} \varphi^{*}\left(U_{t / 2} P U_{t / 2}^{*}: Q\right) d t=\frac{1}{2} \int_{0}^{+\infty} \varphi^{*}\left(U_{t} P U_{t}^{*}: Q\right) 2 d t=2 i^{*}(P: Q) .
$$


First, assume that $P, Q$ are in generic position. By Lemma 4.2, the lefthand side of $(4.5)$ is identical to $-2 \chi_{\mathrm{orb}}(P, Q)$. Thus the desired identity follows. Next, assume that $P, Q$ are not in generic position. By what we have done in Section 3, $\hat{\mu}_{0}$ must have at least one atom at either 0 or $\pi$ with weight $c_{1}(0)-c_{1}(+0) \supsetneqq 0$ or $c_{0}(0)-c_{0}(+0) \supsetneqq 0$, respectively. Thus the left-hand side of (4.5) must be $+\infty$, and therefore, so is $i^{*}(P ; Q)$. By definition $\chi_{\mathrm{orb}}(P, Q)=-\infty$ in this case, and hence the desired identity holds as $+\infty=+\infty$.

In closing, we illustrate how to use the subordination relation in Proposition 3.1 .

Lemma 4.4. If $H(t, \zeta)$ (see Proposition 3.1) defines a function in $\zeta$ of Hardy class $H^{3 / 2}(\mathbb{D})$ (see [19, Section IV.B.2]) for every $t>0$, then $i^{*}(\mathbb{C} P+$ $\mathbb{C}(I-P) ; \mathbb{C} Q+\mathbb{C}(I-Q))=-\chi_{\text {orb }}(P, Q)$ holds.

Proof. Let $L(t, \zeta)$ be as in Section 3 , and write

$$
\begin{aligned}
\tilde{L}(t, \zeta) & :=\int_{(-\pi, \pi]} \frac{e^{\sqrt{-1} \theta}+\zeta}{e^{\sqrt{-1} \theta}-\zeta}\left(\hat{\mu}_{t}+a \delta_{\pi}+b \delta_{0}\right)(d \theta) \\
& =L(t, \zeta)+a \frac{1-\zeta}{1+\zeta}+b \frac{1+\zeta}{1-\zeta} .
\end{aligned}
$$

By the PDE (3.2), one has $\frac{\partial \operatorname{Re} \tilde{L}}{\partial t}(\zeta)=\frac{\partial \operatorname{Re} L}{\partial t}(\zeta)=-\frac{\partial}{\partial \theta}(\operatorname{Re} \tilde{L}(\zeta) \cdot \operatorname{Im} L(\zeta)+$ $\operatorname{Im} \tilde{L}(\zeta) \cdot \operatorname{Re} L(\zeta))$ in $\zeta=r e^{\sqrt{-1 \theta}}$ with $0<r<1$. Write

$$
\Sigma_{s}(f, g):=2 \iint_{(-\pi, \pi]^{2}} \log \left|1-s e^{\sqrt{-1}(\alpha-\beta)}\right| f\left(e^{\sqrt{-1} \alpha}\right) g\left(e^{\sqrt{-1} \beta}\right) \frac{d \alpha}{2 \pi} \frac{d \beta}{2 \pi}
$$

with $0<s<1$ for simplicity. With the Poisson kernel $P_{r}(\theta)$, the same trick as in the proof of [22, Proposition 10.8] shows that

$$
\begin{aligned}
\Sigma_{s}( & \left.P_{r} *\left(\hat{\mu}_{t_{2}}+a \delta_{\pi}+b \delta_{0}\right), P_{r} * \hat{\mu}_{t_{2}}\right)-\Sigma_{s}\left(P_{r} *\left(\hat{\mu}_{t_{1}}+a \delta_{\pi}+b \delta_{0}\right), P_{r} * \hat{\mu}_{t_{1}}\right) \\
= & \frac{1}{2} \int_{t_{1}}^{t_{2}} \int_{(-\pi, \pi]} \operatorname{Im}\left(2 L\left(t, s r e^{\sqrt{-1} \theta}\right)+a \frac{1-s r e^{\sqrt{-1} \theta}}{1+s r e^{\sqrt{-1} \theta}}+b \frac{1+s r e^{\sqrt{-1} \theta}}{1-s r e^{\sqrt{-1} \theta}}\right) \\
& \times \operatorname{Im}\left(2 L\left(t, r e^{\sqrt{-1} \theta}\right)+a \frac{1-r e^{\sqrt{-1} \theta}}{1+r e e^{\sqrt{-1} \theta}}+b \frac{1+r e^{\sqrt{-1} \theta}}{1-r e^{\sqrt{-1} \theta}}\right) \\
& \times 2 \operatorname{Re} L\left(t, r e^{\sqrt{-1} \theta}\right) \frac{d \theta}{2 \pi} d t
\end{aligned}
$$




$$
\begin{aligned}
& +\int_{t_{1}}^{t_{2}}\left[\int_{(-\pi, \pi]} \operatorname{Im}(L+\tilde{L})\left(t, s r e^{\sqrt{-1} \theta}\right) \operatorname{Im} L\left(t, r e^{\sqrt{-1} \theta}\right)\right. \\
& \left.\times \operatorname{Re}\left(a \frac{1-r e^{\sqrt{-1} \theta}}{1+r e^{\sqrt{-1} \theta}}+b \frac{1+r e^{\sqrt{-1} \theta}}{1-r e^{\sqrt{-1} \theta}}\right) \frac{d \theta}{2 \pi}\right] d t
\end{aligned}
$$

for every $0<t_{1}<t_{2}<\infty$.

In what follows, we crucially use the obvious fact that $\operatorname{Re} L(t, \zeta), \operatorname{Re}\left(\frac{1-\zeta}{1+\zeta}\right)$, and $\operatorname{Re}\left(\frac{1+\zeta}{1-\zeta}\right)$ are nonnegative. Since $(\operatorname{Re} L(t, \zeta))^{2} \leq \operatorname{Re} L(t, \zeta) \operatorname{Re} \tilde{L}(t, \zeta) \leq$ $|H(t, \zeta)|$, the assumption here implies that $\hat{\mu}_{t}$ has an $L^{3}$-density $\hat{h}(t, \theta)$, that is, $\hat{\mu}_{t}(d \theta)=\hat{h}(t, \theta) d \theta$, for every $t>0$ (see [19, p. 15]). We fix arbitrary $0<t_{1}<t_{2}<+\infty$ for a while. Set $C_{t_{1}}:=\sup _{r<1}\left\|H\left(t_{1}, r e^{\sqrt{-1}(-)}\right)\right\|_{3 / 2}<+\infty$ by assumption, where $\|-\|_{p}$ denotes the usual $L^{p}$-norm with respect to $d \theta$ rather than $d \theta / 2 \pi$ following [19]. By the subordination relation in Proposition 3.1 with Littlewood's subordination principle (see [10, Theorem 1.7]), one has

$$
\begin{aligned}
& \left\|\operatorname{Re} L\left(t, r e^{\sqrt{-1}(-)}\right)\right\|_{3} \leq\left\|H\left(t, r e^{\sqrt{-1}(-)}\right)\right\|_{3 / 2}^{1 / 2} \leq C_{t_{1}}^{1 / 2} ; \\
& \text { hence }\|\hat{h}(t,-)\|_{3} \leq C_{t_{1}}^{1 / 2} / 2 \pi
\end{aligned}
$$

for every $t \geq t_{1}$ and $0<r<1$. Since $|\operatorname{Im} L(t, \zeta)| \operatorname{Re}\left(a \frac{1-\zeta}{1+\zeta}+b \frac{1+\zeta}{1-\zeta}\right) \leq$ $|\operatorname{Im} L(t, \zeta)| \operatorname{Re} \tilde{L}(t, \zeta) \leq|H(t, \zeta)|$, one has

$$
\begin{aligned}
& \left\|\operatorname{Im} L\left(t, S r e^{\sqrt{-1}(-)}\right) \operatorname{Re}\left(a \frac{1-r e^{\sqrt{-1}(-)}}{1+r e^{\sqrt{-1}(-)}}+b \frac{1+r e^{\sqrt{-1}(-)}}{1-r e^{\sqrt{-1}(-)}}\right)\right\|_{3 / 2} \\
& \quad \leq\left\|H\left(t, r e^{\sqrt{-1}(-)}\right)\right\|_{3 / 2} \leq C_{t_{1}}
\end{aligned}
$$

for every $t \geq t_{1}$ and $0<r<1$, again by the subordination relation in Proposition 3.1 with Littlewood's subordination principle. Using the CauchySchwarz inequality (with respect to $\operatorname{Re}(\cdots) d \theta / 2 \pi d t$ ) and then the Hölder inequality (with respect to $d \theta$ and exponents $3,3 / 2$ ) together with Riesz's theorem (see [19, p. 91]), we see that the absolute value of the second term of the right-hand side of (4.6) is not greater than

$$
\begin{aligned}
& \left\{\int _ { t _ { 1 } } ^ { t _ { 2 } } \left[\int_{(-\pi, \pi]}\left|\operatorname{Im}(L+\tilde{L})\left(t, s r e^{\sqrt{-1} \theta}\right)\right|^{2}\right.\right. \\
& \left.\left.\quad \times \operatorname{Re}\left(a \frac{1-r e^{\sqrt{-1} \theta}}{1+r e^{\sqrt{-1} \theta}}+b \frac{1+r e^{\sqrt{-1} \theta}}{1-r e^{\sqrt{-1} \theta}}\right) \frac{d \theta}{2 \pi}\right] d t\right\}^{1 / 2} C_{t_{1}}^{3 / 4} \sqrt{C^{\prime}\left(t_{2}-t_{1}\right) / 2 \pi}
\end{aligned}
$$


with a universal constant $C^{\prime}>0$ (that comes from Riesz's theorem) and, moreover, that this converges to 0 as $r \nearrow 1$ thanks to [19, pp. 7-8], (4.7), the continuity of $\operatorname{Im}(L+\tilde{L})(t, \zeta)$ in $(t, \zeta)$, and $\operatorname{Im}(L+\tilde{L})(t, \pm s)=0$ (due to $\hat{h}(-\theta)=\hat{h}(\theta))$. Once again by the subordination relation in Proposition 3.1 with Littlewood's subordination principle, we have

$$
\begin{aligned}
& \| \operatorname{Im}\left(2 L\left(t, r e^{\sqrt{-1}(-)}\right)+a \frac{1-r e^{\sqrt{-1}(-)}}{1+r e^{\sqrt{-1}(-)}}+b \frac{1+r e^{\sqrt{-1}(-)}}{1-r e^{\sqrt{-1}(-)}}\right) \\
& \quad \times 2 \operatorname{Re} L\left(t, r e^{\sqrt{-1}(-)}\right) \|_{3 / 2} \\
& \leq 4\left\|H\left(t, r e^{\sqrt{-1}(-)}\right)\right\|_{3 / 2} \leq 4 C_{t_{1}}
\end{aligned}
$$

for every $t \geq t_{1}$ and $0<r<1$, and we can easily confirm, with the help of facts in [19, pp. 9, 88-89], that the first term of the right-hand side of (4.6) converges to

$$
\begin{aligned}
& \frac{1}{2} \int_{t_{1}}^{t_{2}} \int_{(-\pi, \pi]} \operatorname{Im}\left(2 L\left(t, s e^{\sqrt{-1} \theta}\right)+a \frac{1-s e^{\sqrt{-1} \theta}}{1+s e^{\sqrt{-1} \theta}}+b \frac{1+s e^{\sqrt{-1} \theta}}{1-s e^{\sqrt{-1} \theta}}\right) \\
& \quad \times(2 \pi H(2 \hat{h}(t,-))(\theta)-a \tan (\theta / 2)+b \cot (\theta / 2)) 2 \hat{h}(t, \theta) d \theta d t
\end{aligned}
$$

as $r \nearrow 1$. Consequently, we have, for every $0<s<1$,

$$
\begin{gathered}
-\left\{2 \iint_{(-\pi, \pi]^{2}} \log \left|1-s e^{\sqrt{-1}(\alpha-\beta)}\right|\left(\hat{\mu}_{t_{1}}+a \delta_{\pi}+b \delta_{0}\right)(d \alpha) \hat{\mu}_{t_{1}}(d \beta)+Z(s)\right\} \\
=\frac{1}{2} \int_{t_{1}}^{t_{2}} \int_{(-\pi, \pi]} \operatorname{Im}\left(2 L\left(t, s e^{\sqrt{-1} \theta}\right)+a \frac{1-s e^{\sqrt{-1} \theta}}{1+s e^{\sqrt{-1} \theta}}+b \frac{1+s e^{\sqrt{-1} \theta}}{1-s e^{\sqrt{-1} \theta}}\right)
\end{gathered}
$$

$$
\begin{aligned}
& \times(2 \pi H(2 \hat{h}(t,-))(\theta)-a \tan (\theta / 2)+b \cot (\theta / 2)) 2 \hat{h}(t, \theta) d \theta d t \\
& -\left\{2 \iint_{(-\pi, \pi]^{2}} \log \left|1-s e^{\sqrt{-1}(\alpha-\beta)}\right|\right. \\
& \left.\times\left(\hat{\mu}_{t_{2}}+a \delta_{\pi}+b \delta_{0}\right)(d \alpha) \hat{\mu}_{t_{2}}(d \beta)+Z(s)\right\},
\end{aligned}
$$

where $Z(s):=Z_{\tau(P), \tau(Q)}-\frac{1-(a+b)^{2}}{2} \log s(n . b .$, the total mass of the product measure $\left(\hat{\mu}_{t}+a \delta_{\pi}+b \delta_{0}\right) \otimes \hat{\mu}_{t}$ is $\frac{1-(a+b)^{2}}{4}$ for every $t \geq 0$; see Section 3$)$. Write $k(t, \theta):=2 \pi H(2 \hat{h}(t,-))(\theta)-a \tan (\theta / 2)+b \cot (\theta / 2)$ for simplicity. By (4.8) with [19, pp. 9, 88-89], one has

$$
\|k(t,-) 2 \hat{h}(t,-)\|_{3 / 2} \leq 2 C_{t_{1}} / \pi
$$


for every $t \geq t_{1}$. By Lemma 4.1 with the aid of (4.7) and (4.10), and moreover, by [22, Proposition 10.11(a)],

$$
\begin{aligned}
\int_{t_{1}}^{t_{2}} \int_{(-\pi, \pi]} k(t, \theta)^{2} 2 \hat{h}(t, \theta) d \theta d t & =\int_{t_{1}}^{t_{2}} \varphi^{*}\left(U_{t} P U_{t}^{*}: Q\right) d t \\
& \leq 2 i^{*}\left(U_{t_{1}} P U_{t_{1}}^{*}: Q\right)<+\infty .
\end{aligned}
$$

By the Hölder inequality, (4.7), (4.10), and Riesz's theorem,

$$
\int_{t_{1}}^{t_{2}} \int_{(-\pi, \pi]}|2 \pi H(2 \hat{h}(t,-))(\theta)||k(t, \theta)| 2 \hat{h}(t, \theta) d \theta d t<+\infty .
$$

Note that

$$
\begin{aligned}
a|\tan (\theta / 2)| \leq & a \mathbf{1}_{\{|\theta| \leq \pi / 2\}}(\theta) \\
& +|-k(t, \theta)+2 \pi H(2 \hat{h}(t,-))(\theta)+b \cot (\theta / 2)| \mathbf{1}_{\{|\theta|>\pi / 2\}}(\theta) \\
\leq & a \mathbf{1}_{\{|\theta| \leq \pi / 2\}}(\theta) \\
& +(|k(t, \theta)|+|2 \pi H(2 \hat{h}(t,-))(\theta)|+b) \mathbf{1}_{\{|\theta|>\pi / 2\}}(\theta)
\end{aligned}
$$

and similarly that

$$
\begin{aligned}
& b|\cot (\theta / 2)| \\
& \quad \leq b \mathbf{1}_{\{|\theta|>\pi / 2\}}(\theta)+(|k(t, \theta)|+|2 \pi H(2 \hat{h}(t,-))(\theta)|+a) \mathbf{1}_{\{|\theta| \leq \pi / 2\}}(\theta) .
\end{aligned}
$$

Hence

$$
\begin{aligned}
& a\left|\operatorname{Im}\left(\frac{1-s e^{\sqrt{-1} \theta}}{1+s e^{\sqrt{-1} \theta}}\right)\right|+b\left|\operatorname{Im}\left(\frac{1+s e^{\sqrt{-1} \theta}}{1-s e^{\sqrt{-1} \theta}}\right)\right| \\
& \quad \leq a|\tan (\theta / 2)|+b|\cot (\theta / 2)| \\
& \quad \leq 2(a+b)+|k(t, \theta)|+|2 \pi H(2 \hat{h}(t,-))(\theta)|,
\end{aligned}
$$

which is integrable with respect to the measure $|k(t, \theta)| 2 \hat{h}(t, \theta) d \theta d t$ over $(-\pi, \pi] \times\left[t_{1}, t_{2}\right]$. With these remarks and [19, pp. 9, 88-89] again, the dominated convergence theorem (with respect to the measure $|k(t, \theta)| 2 \hat{h}(t, \theta) d \theta d t$ over $\left.(-\pi, \pi] \times\left[t_{1}, t_{2}\right]\right)$ shows that the first term of the right-hand side of (4.9) converges to $\frac{1}{2} \int_{t_{1}}^{t_{2}} \varphi^{*}\left(U_{t} P U_{t}^{*}: Q\right) d t$ as $s \nearrow 1$. By Lemma 4.2 with the aid of the first five lines of $[22$, p. 147], we finally get

$$
-\chi_{\mathrm{orb}}\left(U_{t_{2}} P U_{t_{2}}^{*}, Q\right)+\frac{1}{2} \int_{t_{1}}^{t_{2}} \varphi^{*}\left(U_{t} P U_{t}^{*}: Q\right) d t=-\chi_{\mathrm{orb}}\left(U_{t_{1}} P U_{t_{1}}^{*}, Q\right) .
$$


By [16, Theorem 2.1], [22, Proposition 10.11(a)], and [13, Proposition 4.6], one has

$$
\begin{aligned}
\int_{t_{1}}^{+\infty}-\chi_{\mathrm{orb}}\left(U_{t_{2}} P U_{t_{2}}^{*}, Q\right) d t_{2} & \leq \int_{t_{1}}^{+\infty} \varphi^{*}\left(U_{t_{2}} P U_{t_{2}}^{*}: Q\right) d t_{2} \\
& =2 i^{*}\left(U_{t_{1}} P U_{t_{1}}^{*}: Q\right)<+\infty
\end{aligned}
$$

implying $\lim _{t_{2}} \nearrow_{+\infty} \chi_{\mathrm{orb}}\left(U_{t_{2}} P U_{t_{2}}^{*}, Q\right)=0$. (This trick originates in a preprint version of [16].) By [13, Proposition 2.5(4), Proposition 4.6], one has $\lim _{t_{1} \searrow 0} \chi_{\text {orb }}\left(U_{t_{1}} P U_{t_{1}}^{*}, Q\right)=\chi_{\text {orb }}(P, Q)$. Hence we are done.

By $(3.2), H(t, \zeta)$ becomes the constant $\left(1-(a+b)^{2}\right) / 4$ in the time stationary case; hence the assumption of Lemma 4.4 is not strange. Also, the lemma re-proves Theorem 4.3 completely. In fact, it has been known, essentially due to Biane [2, Lemma 12], [3, Theorem 3.5(4)] thanks to Proposition 3.3, that $L(t, \zeta)=\frac{1}{2}+\int_{(-\pi, \pi]} \frac{e^{\sqrt{-1} \theta} \zeta}{1-e^{\sqrt{-1} \theta} \zeta}\left(2 \hat{\mu}_{t}\right)(d \theta)$ becomes a function in $\zeta$ of Hardy class $H^{\infty}(\mathbb{D})$ for every $t>0$ (and hence so does $H(t, \zeta)=L(t, \zeta)^{2}$ too) when $\tau(P)=\tau(Q)=1 / 2$. Here is a sample application of Lemma 4.4.

Corollary 4.5. Assume that the measure $\mu_{0}$ (see Section 3) has an $L^{3}$ density with respect to $x(1-x) d x$ on $[0,1]$ and is supported in $[\alpha, \beta]$ such that $\alpha \supsetneqq 0$ if $\tau(P) \neq \tau(Q)$ (i.e., $a \neq 0$ ) and $\beta \supsetneqq 1$ if $\tau(P)+\tau(Q) \neq 1$ (i.e., $b \neq 0)$. Then $i^{*}(\mathbb{C} P+\mathbb{C}(I-P) ; \mathbb{C} Q+\mathbb{C}(I-Q))=-\chi_{\text {orb }}(P, Q)$ holds.

Proof. For simplicity, assume both $a=|\tau(P)-\tau(Q)| \neq 0$ and $b=\mid \tau(P)+$ $\tau(Q)-1 \mid \neq 0$. Since $\mu_{0}$ has an $L^{3}$-density with respect to $x(1-x) d x$ on $(0,1)$, one easily sees that $\hat{\mu}_{0}$ has an $L^{3}$-density $\hat{h}(0, \theta)$ (with respect to $d \theta$ ); hence $L(0, \zeta)$ is a function in $\zeta$ of Hardy class $H^{3}(\mathbb{D})$ by Riesz's theorem with a standard fact (see [19, pp. 9, 88-89]). Moreover, the assumption on the support of $\mu_{0}$ immediately implies that $L(0, \zeta)$ has analytic continuation across both points $\zeta= \pm 1$ to the outside of $\overline{\mathbb{D}}$. Since $\lim _{\zeta \rightarrow \pm 1} L(0, \zeta)=0$, $L(0, \zeta)$ admits a power series expansion without constant term around $\zeta=$ \pm 1 . Thus $\left(a \frac{1-\zeta}{1+\zeta}+b \frac{1+\zeta}{1-\zeta}\right) L(0, \zeta)$ is bounded in some neighborhoods at both $\zeta= \pm 1$. With these remarks, it is plain to see that $H(0, \zeta)$ is a function in $\zeta$ of Hardy class $H^{3 / 2}(\mathbb{D})$. Hence the subordination relation in Proposition 3.1 with Littlewood's subordination principle (see [10, Theorem 1.7]) implies that $H(t, \zeta)$ becomes a function in $\zeta$ of Hardy class $H^{3 / 2}(\mathbb{D})$ for every $t \geq 0$ so that the desired assertion holds thanks to Lemma 4.4. 
The above fact suggests that the question should be answered in the affirmative without assuming that $\tau(P)=\tau(Q)=1 / 2$. The only missing piece in our attempt is apparently a more detailed study of $H(t, \zeta)$ and/or the conformal transformations $f_{t}(\zeta)$; thus the question comes down to a study of Loewner-Kufarev equations. We should probably point out here that Lemma 4.1 (an explicit computation of the liberation gradient) uses the assumption (4.3) so that [22, Corollary 8.6] (a regularity result via liberation) does not imply the "necessary regularity" of $\hat{\mu}_{t}$ for $t>0$.

Finally, we remind the reader that [13, Proposition 6.3] shows that $\chi_{\mathrm{orb}}\left(U_{t} P U_{t}^{*}, Q\right)$ is finite for every $t>0$. Hence the measure $\mu_{t}$ has no atom, and $U_{t} P U_{t}^{*}, Q$ must be in generic position for every $t>0$ thanks to, for example, [16, Lemma 1.1] and [13, Lemma 2.4]. This gives another proof to (a part of) Remark 3.5 and also shows that all the von Neumann algebras $W^{*}\left(U_{t} P U_{t}^{*}, Q\right), t>0$, are isomorphic.

Acknowledgments. We thank Fumio Hiai for discussions on this subject matter and comments to a draft of the present notes. We also thank one of the referees for comments to these notes. The first author's work was partially supported by JSPS Grant-in-Aid for Scientific Research (B) 22340032. The second author's work was partially supported by JSPS Grant-in-Aid for Scientific Research (C) 24540214.

\section{REFERENCES}

[1] Ph. Biane, "Free Brownian motion, free stochastic calculus and random matrices" in Free Probability Theory (Waterloo, Canada, 1995), Fields Inst. Commun 12, Amer. Math. Soc., Providence, 1997, 1-19. MR 1426833.

[2] — Segal-Bargmann transform, functional calculus on matrix spaces and the theory of semi-circular and circular systems, J. Funct. Anal. 144 (1997), 232-286. MR 1430721. DOI 10.1006/jfan.1996.2990.

[3] — Processes with free increments, Math. Z. 227 (1998), 143-174. MR 1605393. DOI $10.1007 /$ PL00004363.

[4] Ph. Biane and Y. Dabrowski, Concavification of free entropy, Adv. Math. 234 (2013), 667-696. MR 3003941. DOI 10.1016/j.aim.2012.11.003.

[5] Ph. Biane and R. Speicher, Stochastic calculus with respect to free Brownian motion and analysis on Wigner space, Probab. Theory Related Fields 112 (1998), 373-409. MR 1660906. DOI 10.1007/s004400050194.

[6] B. Collins and T. Kemp, Liberation of projections, J. Funct. Anal. 266 (2014), 19882052. MR 3150150. DOI 10.1016/j.jfa.2013.10.034.

[7] Y. Dabrowski, A free stochastic partial differential equation, Ann. Inst. Henri Poincaré Probab. Stat. 50 (2014), 1404-1455. MR 3270000. DOI 10.1214/13-AIHP548.

[8] N. Demni, Free Jacobi process, J. Theoret. Probab. 21 (2008), 118-143. MR 2384475. DOI 10.1007/s10959-007-0110-1. 
[9] N. Demni, T. Hamdi, and T. Hmidi, Spectral distribution of the free Jacobi process, Indiana Univ. Math. J. 61 (2012), 1351-1368. MR 3071702. DOI 10.1512/iumj.2012.61.5034.

[10] P. L. Duren, Theory of $H^{p}$ Spaces, Dover, New York, 2000.

[11] Ya. L. Geronimus, Polynomials orthogonal on a circle and their applications, Amer. Math. Soc. Transl. Ser. 21954 (1954), 79 pp. MR 0061706.

[12] P. R. Halmos, A Hilbert Space Problem Book, 2nd ed., Grad. Texts in Math. 19, Springer, New York, 1982. MR 0675952.

[13] F. Hiai, T. Miyamoto, and Y. Ueda, Orbital approach to microstate free entropy, Internat. J. Math. 20 (2009), 227-273. MR 2493361. DOI 10.1142/S0129167X09005261.

[14] F. Hiai and D. Petz, Large deviations for functions of two random projection matrices, Acta Sci. Math. (Szeged) 72 (2006), 581-609. MR 2289756.

[15] F. Hiai and Y. Ueda, Notes on microstate free entropy of projections, Publ. Res. Inst. Math. Sci. 44 (2008), 49-89. MR 2405867.

[16] — A log-Sobolev type inequality for free entropy of two projections, Ann. Inst. Henri Poincaré Probab. Stat. 45 (2009), 239-249. MR 2500237. DOI 10.1214/08-AIHP164.

[17] V. Kargin, On free stochastic differential equations, J. Theoret. Probab. 24 (2011), 821-848. MR 2822483. DOI 10.1007/s10959-011-0341-z.

[18] F. W. King, Hilbert Transforms, Vol. 1, Encyclopedia Math. Appl. 124, Cambridge University Press, Cambridge, 2009. MR 2542214.

[19] P. Koosis, Introduction to $H_{p}$ Spaces, 2nd ed., with two appendices by V. P. Havin, Cambridge Tracts in Math. 115, Cambridge University Press, Cambridge, 1998. MR 1669574.

[20] G. F. Lawler, Conformally Invariant Processes in the Plane, Math. Surveys Monogr. 114, Amer. Math. Soc., Providence, 2005. MR 2129588.

[21] Y. Ueda, Orbital free entropy, revisited, Indiana Univ. Math. J. 63 (2014), 551-577. MR 3233219. DOI 10.1512/iumj.2014.63.5220.

[22] D. Voiculescu, The analogues of entropy and of Fisher's information measure in free probability theory, VI: Liberation and mutual free information, Adv. Math. 146 (1999), 101-166. MR 1711843. DOI 10.1006/aima.1998.1819.

[23] - "Lectures on free probability theory" in Lectures on Probability Theory and Statistics (Saint-Flour, 1998), Lecture Notes in Math. 1738, Springer, Berlin, 2000, 279-349. MR 1775641. DOI 10.1007/BFb0106703.

[24] — Free entropy, Bull. Lond. Math. Soc. 34 (2002), 257-278. MR 1887698. DOI 10.1112/S0024609301008992.

[25] P. Zhong, On the free convolution with a free multiplicative analogue of the normal distribution, to appear in J. Theor. Probab., preprint, arXiv:1211.3160v3 [math.PR]. 
Masaki Izumi

Department of Mathematics Graduate School of Science

Kyoto University

Sakyo-ku, Kyoto 606-8502

Japan

izumi@math.kyoto-u.ac.jp

Yoshimichi Ueda

Graduate School of Mathematics

Kyushu University

Fukuoka, 819-0395

Japan

ueda@math.kyushu-u.ac.jp 